10. Perelman AH. Management of hyperthyroidism and thyroid storm during pregnancy. In: Foley MR, Strong TH. (editors). Obstetric intensive care. Philadelphia: WB Saunders; 1997. p. 147-57.

11. Avila WS, Rossi EG, Ramires JAF, Grinberg M, Bortolotto MRL, Zugaib M, et al. Pregnancy in patients with heart disease: experience with 1000 cases. Clin Cardiol. 2003; 26: 135-42.

12. Sociedade Brasileira de Cardiologia. I Diretriz Latino-Americana para avaliação e conduta na insuficiência cardíaca descompensada. Arq Bras Cardiol. 2005; 85 (supl. 3): 1-94

13. Mestman JH. Hypothyroidism and hyperthyroidism. In: Queenan JT. (editor) Management of high-risk pregnancy. 4th ed. Malden: Blackwell Science; 1999. p.271-3.

14. Austin JHM. Postpartum pleural effusion. Ann Intern Med. 1983; 98: 555-6.

15. Sociedade Brasileira de Cardiologia. II Diretrizes para o diagnóstico e tratamento da insuficiência cardíaca. Arq Bras Cardiol. 2002; 79: 1-30.

16. Elkayam U, Gleicher N. Cardiac evaluation during pregnancy. In: Elkayam $\mathrm{U}$, Gleicher N. (editors). Cardiac problems in pregnancy. 3rd ed. New York: Wiley-Liss; 1998. p.23-32.

17. Andrade MM, Pontes S Jr. Avaliação ecocardiográfica da gestante cardíaca. In: Andrade J, Avila WA. (editores). Doença cardiovascular, gravidez e planejamento familiar. São Paulo: Atheneu; 2003. p. 25-30.

18. Dorbala S, Brozena S, Zeb S, Galatro K, Homel P, Ren JF, et al. Risk stratification of women with peripartum cardiomyopathy at inicial presentation: a dobutamine stress echocardiography study. J Am Soc Echocardiogr. 2005; 18: $45-8$.

19. Mabie CW. Basic hemodynamic monitoring for the obstetric care provider. In

\section{Tromboembolismo venoso agudo}

\section{Marcelo Basso Gazzana, Citânia Lúcia Tedoldi, Sérgio Saldanha Menna Barreto}

\section{1 - Introdução}

A gestação e o período pós-parto são fatores de risco adquiridos para tromboembolia venosa (TEV) aguda, que consiste em duas condições interrelacionadas: trombose venosa profunda (TVP) e tromboembolismo pulmonar (TEP) ${ }^{1,2}$. Considerando as dificuldades de diagnóstico e a presença de casos assintomáticos, a incidência verdadeira de TEP na gravidez é desconhecida. A maioria das TVP são ileofemorais (72\%), mais propensas a embolizar para o pulmão ${ }^{3}$. A incidência de TEV é maior no terceiro trimestre e no puerpério, assim como no parto cesáreo (sobretudo se realizado de urgência), quando comparado ao parto vaginal ${ }^{3,4}$.

O TEP é a primeira causa de morte materna direta em países desenvolvidos ${ }^{5}$. Sendo a TEV uma condição prevenível e tratável, a mortalidade materna por TEP pode ser reduzida de duas formas:

1) Investigando agressivamente os casos com suspeita de TEV;

2) Fazendo a profilaxia das pacientes sob risco de $\mathrm{TEV}^{6}$.

$\mathrm{O}$ aumento do risco de TEV na gestação deve-se à combinação de modificações hormonais, mecânicas e na composição do sangue favorecendo a trombose, retornando ao normal em 2-6 semanas após o parto ${ }^{7,8}$.
Foley MR, Strong TH. (editors). Obstetric intensive care: a practical manual. Philadelphia: WB Saunders; 1997. p. 6-19.

20. Burwell and Metcalfe's: heart disease and pregnancy-physiology and management. In: Metcalfe J, McAnulty JH, Ueland K. (editors). 2nd ed. Boston: Little Brown and Company; 1986. p. 83-132.

21. Clark SL, Cotton DB, Hankins GDV, Phelan JP. (editors). Handbook of critical care obstetrics. Boston: Blackwell SP; 1994. p. 47-53.

22. Andrade J, Lopes CMC. Uso de medicamentos cardiovasculares na gravidez, parto e puerpério. Rev Soc Cardiol Estado Sao Paulo. 1994; 4: 581-8.

23. Widerhorn J, Rubin JN, Frishmann WH, Elkayam U. Cardiovascular drugs in pregnancy. Cardiol Clin. 1987; 5: 651-74

24. Briggs GG, Freeman RK, Yaffe SJ. (editors). Drugs in pregnancy and lactation. 6th ed. Philadelphia: Lippincot Williams \& Wilkins; 2002.

25. Swedberg K, Cleland J, Dargie H, Drexler H, Follath F, Kamayda M, et al. The Task Force for the diagnosis and treatment of CHF of the European Society of Cardiology. Eur Heart J. 2005; 26: 1115-40.

26. Pereira Barreto AC, Bocchi EA. (editores). Insuficiência cardíaca. São Paulo: Segmento; 2003.

27. Bates SM, Greer IA, Hirsh J, Ginsberg JS. Use of antithrombotic agents during Pregnancy. Chest. 2004; 126: 627S-644S.

28. Hunt SA, Abrahan W, Chin MH, Feldman AM, Francis GS, Ganiats TG, et al. ACC/AHA Guideline update for the diagnosis and management of chronic heart failure in the adult: summary article. Circulation. 2005; 112: e154-235.

29. Moreira DAR. Uso de medicamentos antiarrítmicos durante a gravidez. Rev Soc Cardiol Estado Sao Paulo. 1998; 6: 1146-56.

Há vários fatores de risco estabelecidos para TEV, embasados em estudos em gestantes e não gestantes (Tabela 1).

Anormalidades trombofílicas, como deficiência de proteína C, proteína S e antitrombina, aumentam o risco de trombose em 10 vezes durante a gestação, sendo a de maior risco a deficiência de antitrombina. Entre as trombofilias genéticas mais frequentes, o fator $\mathrm{V}$ Leiden (prevalência de $5 \%$ na população normal e de $20 \%$ em pacientes com trombose), quando heterozigótico, aumenta o risco de TEV em 3-8 vezes, e quando homozigótico, em 50-80 vezes. A mutação da protrombina, presente em $2-3 \%$ da população e em $6 \%$ de pacientes com trombose, aumenta o risco em três vezes pelo aumento dos níveis da protombina. Essas mutações, quando heterozigóticas, são consideradas fracos fatores de risco e aumentam a incidência de trombose quando associadas, ou em associação com outros fatores de risco ${ }^{9}$.

\section{2 - Diagnóstico da TEV}

O diagnóstico clínico de TVP ou de TEP não é confiável. É fundamental estabelecer um diagnóstico objetivo por meio de exames de imagem. A utilização de exames diagnósticos que produzem radiação, com as precauções adequadas, fazem com que a quantidade absorvida pelo feto e seu consequente risco sejam mínimos ${ }^{10}$.

\subsection{1 - Diagnóstico da TVP}

A maioria das gestantes com edema de membros inferiores (com ou sem dor na panturrilha) no terceiro trimestre não 
Tabela 1 - Fatores de risco para TEV aguda

\begin{tabular}{|c|c|}
\hline Idade materna elevada (> 35 anos) & Imobilidade (> 4 dias) \\
\hline Cateteres venosos centrais & AVC (com paralisia) \\
\hline Obesidade & Trauma/Fraturas \\
\hline TEV prévia & Insuficiência cardíaca \\
\hline Veias varicosas & Cirurgia maior \\
\hline Doença inflamatória intestinal & Uso de estrógeno \\
\hline Câncer/Quimioterapia & Síndrome nefrótica \\
\hline Trombofilias & Hiperemese, desidratação \\
\hline $\begin{array}{l}\text { Trombocitopenia induzida pela } \\
\text { heparina }\end{array}$ & Hiper-homocisteinemia \\
\hline Doenças mieloproliferativas* & Tabagismo \\
\hline Fatores fisiológicos & Fatores nosológicos \\
\hline Hipercoagulabilidade da gestação & Parto instrumentado (uso de fórcipe) \\
\hline Aumento dos fatores de coagulação** & Cesariana (sobretudo de emergência) \\
\hline Diminuição da proteína S & Multiparidade ( $\geq 4$ gestações) \\
\hline Redução da fibrinólise & Estase venosa \\
\hline \multirow[t]{4}{*}{ Aumento da capacitância venosa } & Hemorragia periparto \\
\hline & Inibidores da lactação \\
\hline & Infecções/Doenças intercorrentes \\
\hline & $\begin{array}{l}\text { Procedimentos (curetagem uterina, } \\
\text { esterilização pós-parto) }\end{array}$ \\
\hline
\end{tabular}

*Policitemia vera, trombocitemia essencial, hemoglobinúria paroxística noturna. ${ }^{* *}$ Aumento de fatores de coagulação I, VII, VIII, IX, X e do fator Von Willebrand. Adaptado de Chest, 2001, Bazzan M, et al, $2001^{14}$ e Guideline Royal College of Obstetricians and Gynecologists, 37-200432.

tem TVP. No entanto, se tais sintomas ocorrerem no primeiro trimestre, particularmente na perna esquerda, a presença de TVP é mais provável ${ }^{10}$. Dor em abdome inferior pode ser devido à circulação colateral ou à trombose periovariana ${ }^{3}$. A probabilidade clínica pré-teste é estimada com base na presença de sintomas e sinais, fatores de risco para TEV e possibilidade de um diagnóstico alternativo, classificando as pacientes em alto, intermediário ou baixo risco.

Os testes diagnósticos podem ser não invasivos (ultrassom [US] por compressão com ou sem Doppler, venografia por tomografia computadorizada [TC], ressonância nuclear magnética [RNM]) ou invasivos (flebografia por contraste).

O exame não invasivo recomendado inicialmente é o US por compressão, que deve ser em todo o sistema venoso proximal ${ }^{10}$. Se a suspeita clínica é alta, mas os testes não invasivos são negativos para TVP, a venografia limitada com proteção abdominal que resulta em exposição feral $<0,05$ rads deve ser considerada ${ }^{11}$. Se o resultado do US é duvidoso (pequena área de não compressibilidade), a venografia ou a RNM devem ser solicitadas. A RNM venosa parece ser acurada para o diagnóstico de TVP. É sensível para todos os tipos (incluindo panturrilha, ileofemoral e pelve) e não é associada à exposição radioativa ${ }^{10}$. Pode ser útil para detectar TVP em veias ilíacas, pélvicas ou ovarianas ${ }^{12}$.
Os níveis de D-dímeros plasmáticos aumentam fisiologicamente durante a gestação ${ }^{13}$ e a proporção de pacientes com D-dímeros normais é pequena, além de estarem elevados também na pré-eclâmpsia, descolamento de placenta e parto pré-termo ${ }^{14}$. Se os resultados de US e D-dímeros forem normais, o diagnóstico de TVP sintomática é improvável ${ }^{10}$.

Se o US por compressão é normal na suspeita de TVP de panturrilha, esta não pode ser excluída. Ou indica-se US seriado (repetido em 2-3 dias e em uma semana), ou venografia limitada à panturrilha ${ }^{10}$.

Tromboflebite puerperal de veia ovariana tem incidência em 0,05-0,18\% das gestações. O mecanismo provável é a lesão endotelial seguida de invasão bacteriana. É mais frequente do lado direito. Pode ocorrer extensão para veia cava inferior, ilíaca, renal ou uterina. Essa síndrome é mais comum após o uso de fórcipe e cesariana. Os sintomas são: dor em quadrante inferior direito, febre, náuseas e vômitos. Embolia sistêmica ocorre em 32-38\% dos casos. Diagnóstico diferencial inclui apendicite e abscesso intra-abdominal. O diagnóstico na maioria dos casos tem sido feito por laparotomia ${ }^{8,15}$.

Em 1-2\% das fertilizações in vitro pode ocorrer aumento do risco de TEV em sítios não usuais (sistema nervoso central, membros superiores) e muitas vezes há trombofilia subjacente, sobretudo fator $\mathrm{V}$ Leiden $^{16}$.

O esquema de investigação da TVP na gestação é apresentado na Figura 1.

\subsection{2 - Diagnóstico do tromboembolismo pulmonar} (TEP)

Os sintomas mais comuns são dispneia (62\%), dor pleurítica (46\%) e dor torácica não pleurítica (19\%). Os diagnósticos alternativos mais comuns são pneumonia ou bronquite (41\%) e exacerbação de asma (14\%) ${ }^{17}$. Exames diagnósticos básicos (raio-X de tórax, ECG e gasometria arterial) têm as mesmas limitações que fora da gestação. A gasometria arterial deve ser coletada em posição ereta, já que a $\mathrm{PaO}_{2}$ pode ser até 15 $\mathrm{mmHg}$ menor na posição supina no terceiro trimestre ${ }^{18}$. Após realizar raio-X de tórax para verificar a presença de diagnósticos alternativos, se a suspeita é mantida, deve ser realizado US bilateral de membros inferiores. Se TVP é diagnosticada, o tratamento anticoagulante é iniciado. Se for negativa, deve ser realizada a cintilografia pulmonar perfusional ou a angiotomografia (angioTC) pulmonar. A cintilografia acarreta um aumento da probabilidade de câncer no neonato três vezes maior que a angioTC, enquanto esta acarreta aumento de câncer de mama materno ${ }^{19}$. Quando o raio-X de tórax está alterado, é preferível a opção por angioTC. Se a cintilografia perfusional é normal, não há necessidade de cintilografia ventilatória (o que reduz a exposição fetal à radiação). Se a cintilografia ou angioTC não são diagnósticas e a probabilidade clínica (estimada subjetivamente) é alta ou intermediária, é necessária uma arteriografia. O uso de contraste iodado implica em avaliação funcional tireoidiana no neonato ${ }^{19}$. Se há suspeita clínica persistente de TEP na presença de testes negativos ou com baixa probabilidade, o tratamento deve ser iniciado e os testes diagnósticos repetidos dentro de 7 dias, podendo a terapia ser descontinuada se os exames 


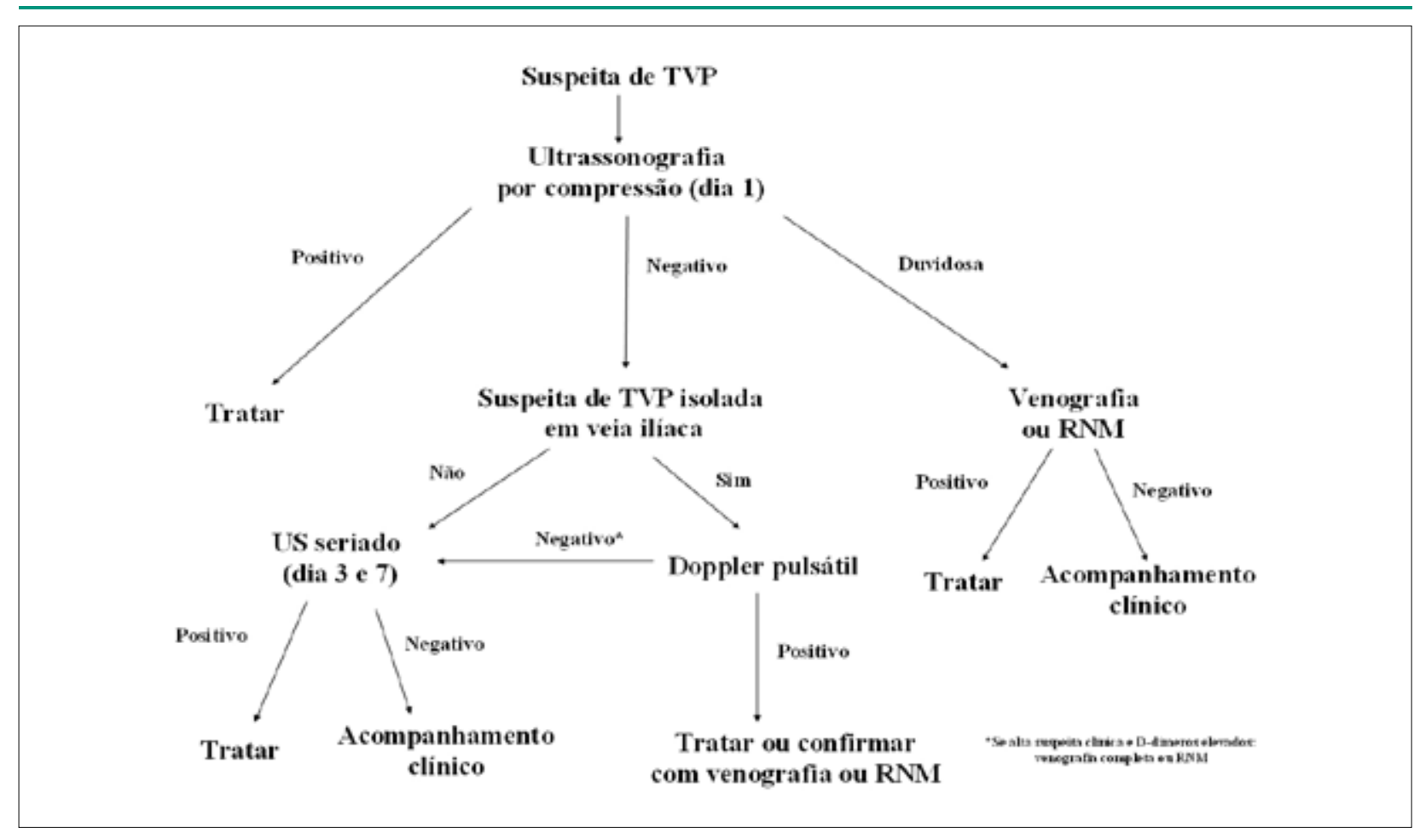

Fig. 1 - Algoritmo de diagnóstico de TVP na gestação.

permanecerem negativos ${ }^{3}$. Após testes negativos, optandose pela suspensão da anticoagulação, as pacientes devem permanecer em acompanhamento clínico rigoroso, já que o valor preditivo das condutas propostas é desconhecido.

O esquema de investigação da TEP na gestação é apresentado na Figura 2.

\section{3 - Tratamento farmacológico}

Há três possibilidades de tratamento anticoagulante: uso de heparina não fracionada (HNF), heparina de baixo peso molecular (HBPM) ou anticoagulante oral (ACO).

A HBPM tem sido preferida em relação à HNF por maior biodisponibilidade e menor risco de trombocitopenia e osteoporose. Como a meia-vida diminui com o decorrer da gestação, é preferível o uso duas vezes ao dia, ajustando a dose de acordo com o peso da paciente, ou monitorando o efeito com antifator Xa coletado $3 \mathrm{~h}$ a $4 \mathrm{~h}$ após a dose matinal, mantendo resultado entre $0,5-1,2 \mathrm{U} / \mathrm{ml}$. Se a opção for pela HNF, deve-se monitorar o efeito pelo TTPA coletado 6 horas após a injeção SC, mantendo em 1,5 a 2,5 vezes o controle basal ${ }^{20,21}$. Quando a escolha é pelo HNF IV, utiliza-se inicialmente bolus de $5.000 \mathrm{UI}(80 \mathrm{UI} / \mathrm{kg})$, seguidos de infusão contínua de, ao menos, $30.000 \mathrm{UI} / 24$ horas para atingir níveis de anticoagulação plena ${ }^{22}$. As heparinas, quando utilizadas por via SC, devem ser descontinuadas 24 horas antes do parto e reiniciadas 12 horas após. Em casos com grande risco de recorrência (TVP proximal ocorrida nas últimas duas semanas), é preferível o uso de HNF IV em doses ajustadas, ocorrendo a suspenção de 4-6 horas antes do parto para reduzir o tempo sem a proteção anticoagulante ${ }^{20}$. Nos partos espontâneos, recomenda-se monitorar o TTPA e usar sulfato de protamina a fim de reduzir o risco de sangramento, lembrando que níveis normais de TTPA podem ocorrer concomitantemente aos níveis elevados de heparina ${ }^{23}$.

Se ocorrer trombocitopenia (contagem de plaquetas < 100.000 ou redução de $50 \%$ em relação ao basal), aumenta o risco de trombose disseminada, que pode ser fatal. A heparina deve ser suspensa e outro tratamento alternativo iniciado (danaparoide sódico ${ }^{10}$, hirudina ${ }^{24}$ ).

Os anticoagulantes orais, como hidroxicumarina, varfarina e femprocumona, atravessam a barreira placentária e são contraindicados para uso durante a embriogênese ( $6^{\underline{a}}$ a $12^{\text {a }}$ semana de gestação) por provocarem embriopatia varfarínica em $6,4 \%$ dos $\operatorname{casos}^{20}$, sendo liberado o seu uso na amamentação ${ }^{25}$. Como o fígado do feto é imaturo, os fatores de coagulação vitamina-K dependentes são baixos, predispondo à anticoagulação excessiva e ao risco de sangramento. Resulta em maior risco de perda perinatal pelo trauma da passagem pelo canal de parto, quando o feto ainda está sob efeito anticoagulante ${ }^{20}$. Sendo assim, os ACO podem ser administrados no segundo e no início do terceiro trimestre em casos selecionados, quando o uso prolongado e de altas doses de heparina é contraindicado.

\subsection{1 - Esquemas de tratamento ${ }^{19,20}$}

a) HNF IV por 5 dias, seguida por HNF SC ou HBPM SC, em doses ajustadas, até 6 semanas pós-parto; ou (IC)

b) HNF SC ou HBPM SC, em doses ajustadas, do início ao final do tratamento (IC). 


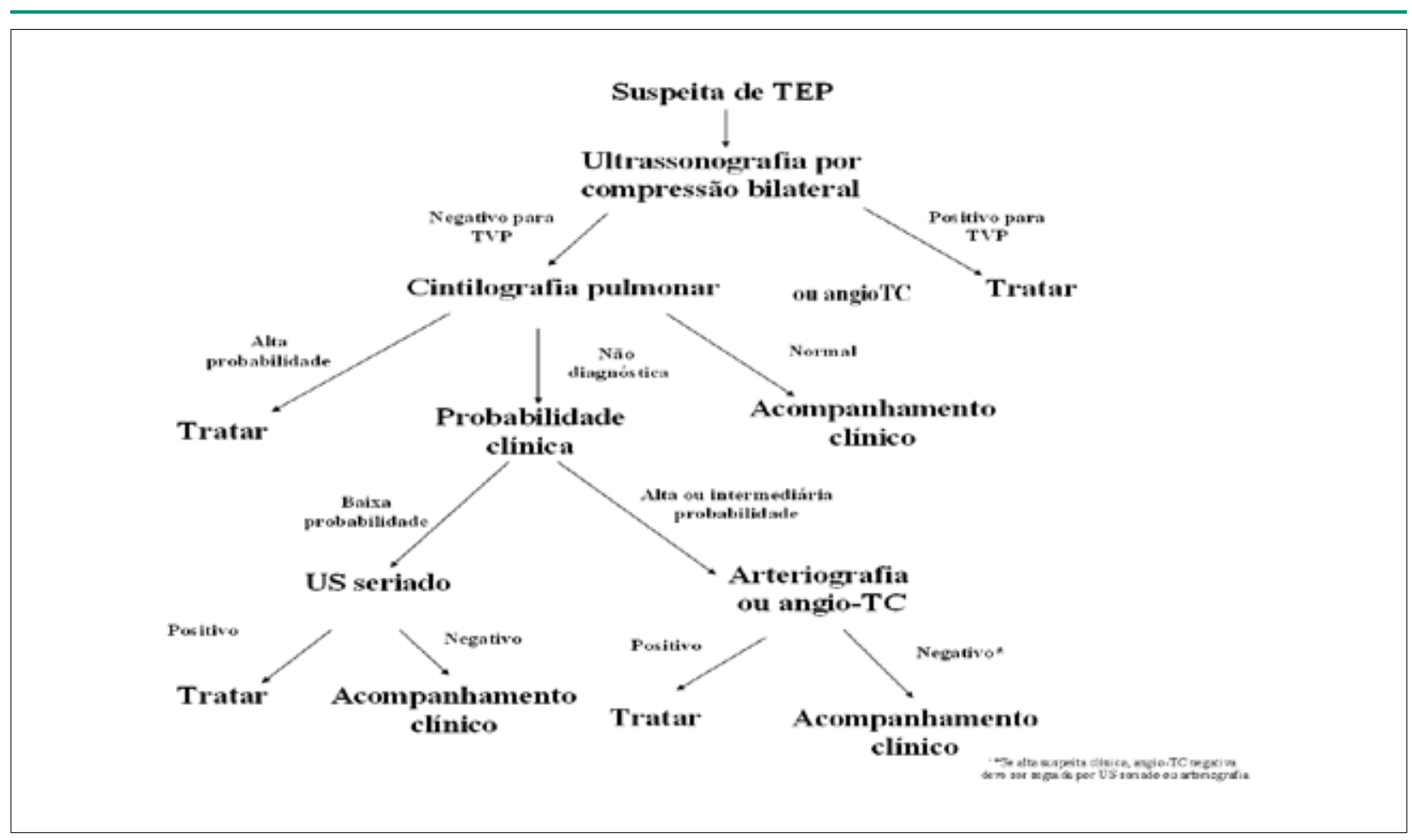

Fig. 2 - Esquema de investigação da TEP na gestação.

c) HNF IV ou SC, ou HBPM SC, em doses ajustadas por 5-7 dias, seguida por ACO (INR entre 2-3) até duas semanas antes do parto (35-36 semanas de gestação), retornando para uma das heparinas até o parto. Reiniciar com HNF ou HBPM, seguida por ACO ou mantendo uma das heparinas até 6 semanas pós-parto (Ila $\mathrm{C}$ ).

d) Alguns clínicos têm mantido tratamento até 12 semanas após o parto por relatos de morte materna devido à TEV entre 6 e 12 semanas $^{26}$. Não há contraindicação em manter a amamentação com o uso de qualquer tipo de anticoagulante, devendo a mesma ser estimulada ${ }^{20}$.

\subsection{2 - Trombolíticos}

A indicação de trombolíticos restringe-se aos casos com instabilidade hemodinâmica, sem resposta ao tratamento de suporte, e quando não há disponibilidade/experiência com embolectomia cirúrgica ${ }^{27,28}$. A maior experiência é com estreptoquinase, sendo poucos casos relatados com uroquinase e alteplase. Não atravessam a barreira placentária, mas podem produzir sangramento materno se utilizados no período periparto. A estreptoquinase, apesar de ter meia-vida curta (35 minutos), está associada a uma demora de 36h-48h para normalizar os níveis de fibrinogênio ${ }^{29}$, período em que o risco de hemorragia é maior.

\subsection{3 - Medidas não farmacológicas}

Os filtros de veia cava têm sido usados na gestação ${ }^{30}$.
As indicações são idênticas às das pacientes não gestantes: contraindicação ou complicações com uso de anticoagulantes ou TEV recorrente, apesar do uso adequado de anticoagulantes. O filtro deve ser colocado em posição suprarrenal, já que a veia ovariana esquerda desemboca na veia renal esquerda ${ }^{22,30,31}$. Os filtros temporários são uma opção em mulheres com contraindicações transitórias à anticoagulação. Após esse período (uma a duas semanas), a anticoagulação é reiniciada e o filtro removido ${ }^{10}$.

Nas pacientes instáveis, quando a trombólise é contraindicada, a embolectomia é a última opção ${ }^{7,32}$. O risco operatório materno é aceitável, mas a taxa de morte fetal é alta $(20 \%$ a $40 \%)$.

Resumindo:

- Em gestantes com suspeita clínica de TEP é fundamental um diagnóstico preciso, pela necessidade de uso prolongado de anticoagulante.

- Os métodos diagnósticos, incluindo a angioTC, podem ser utilizados sem maior risco para o feto. A quantidade de radiação considerada prejudicial para o feto seria $\geq$ a 50.000 $\mu \mathrm{Gy}$ ou $50 \mathrm{mSv}$.

- As HBPM são recomendadas para o tratamento e os ACO devem ser evitados no primeiro e na segunda metade do terceiro trimestre, devendo ser usados com cautela nos demais períodos.

- O tratamento deve ser mantido pelo menos até três meses após o parto ${ }^{33}$. 


\section{Referências}

1. Anderson BS, Steffensen FH, Sorensen HT, Nielson GL, Olsen J. The cumulative incidence of venous thromboembolism during pregnancy and puerperium: an 11 year Danish population based study of 63.300 pregnancies. Acta Obstet Gynecol Scand. 1998; 77: 110-3.

2. Heit JA, Silverstein MD, Mohr DN, Petterson TM, Lohse CM, O'Fallon WN, et al. The epidemiology of venous thromboembolism in the community. Thromb Haemost. 2001; 86: 452-63.

3. Greer IA. Thrombosis in pregnancy: maternal and fetal issues. Lancet. 1999; 353: 1258-65.

4. Eldor A. The use of low-molecular-weight heparin for the management of venous thromboembolism in pregnancy. Eur J Obstet Gynecol Reprod Biol. 2002; 104: 3-13.

5. Bonnar J. Can more be done in obstetric and gynecologic practice to reduce morbidity and mortality associated with venous thromboembolism? Am J Obst Gynecol. 1999; 180: 784-91.

6. Ginsberg JS, Greer I, Hirsh J. Use of antithrombotic agents during pregnancy. Chest. 2001; 119: 122S-131S.

7. ACOG. Practice bulletins. Thromboembolism in pregnancy. Int J Gynecol Obstet. 2001; 75: 203-12.

8. Chan WS, Ginsberg JS. Management of venous thromboembolism in pregnancy. In: Oudkerk M, Van Beek EJR, Ten Cate JW. (editors). Pulmonary embolism. Berlin: Blackwell science; 1999. p. 430-41.

9. Rosendaal FR. Venous thrombosis: the role of genes, environment, and behavior. Hematology Am Soc Hematol Educ Program. 2005; 1-12.

10. Bates SM, Ginsberg JS. How we manage venous thromboembolism during pregnancy. Blood. 2002; 100: 3470-8.

11. Ginsberg JS, Hirsh J, Rainbow AJ, Coates G. Risks to the fetus of radiologic procedures used in the diagnosis of maternal venous thromboembolic disease. Thromb Haemost. 1989; 61: 189-96.

12. Spritzer CE, Evans AC, Kay HH. Magnetic resonance imaging of deep venous thrombosis in pregnant women with lower extremity edema. Obstet Gynecol. 1995; 85: 603-7.

13. Francalanci T, Comeglio P, Liotta AA, Cellai AP, Fedi S, Parretti E, et al. D-dimer concentrations during normal pregnancy, as measured by ELISA. Thromb Res. 1995; 78: 399-405.

14. Proietti AB, Johnson MJ, Proietti FA, Repke JT, Bell WR. Assessment of fibrin (nogen) degradation products in preeclampsia using immunoblot enzymelinked immunosorbent assay, and latex-bead agglutination. Obstet Gynecol. $1991 ; 77: 696-700$.

15. Witlin AG, Sibai BM. Postpartum ovarian vein thrombosis after vaginal delivery: a report of 11 cases. Obstet Gynecol. 1995; 85: 775-80.

16. Aurousseau M, Samama M, Belhasseb A, Herve F, Hugues J. Risk of thromboembolism in relation to an in-vitro fertilisation programme: three case reports. Hum Reprod. 1995; 10: 94-7.

\section{Hipertensão arterial pulmonar}

Marlene Rau de Almeida Callou, Paulo Roberto Miranda Ramos

\section{1 - Introdução}

A hipertensão arterial pulmonar (HAP) é definida como uma anormalidade circulatória caracterizada por aumento da resistência vascular na pequena circulação, em geral por meio de mecanismos mistos, envolvendo vasoconstricção, remodelamento da parede arterial e trombose in situ ${ }^{1}$. O aumento progressivo da resistência vascular pulmonar (RVP) leva à insuficiência ventricular direita (IVD) e morte precoce².
17. Chan WS, Ray JG, Murray S, Coady GE, Coates G, Ginsberg JS. Suspected pulmonary embolism in pregnancy: clinical presentation, results of lung scanning, and subsequent maternal and pediatric outcomes. Arch Intern Med. 2002; 162: 1170-5.

18. Ang CK, Tan TH, Walters WA, Wood C. Postural influence on maternal capillary oxygen and carbon dioxide tension. BMJ. 1969; 4: 201-3.

19. Royal College of Obstetricians and Gynaecologists. Green Top Guideline 28, 2007. Thromboembolic disease in pregnancy and the puerperium: acute management. London: Blackwell; 2007. p. 1-17.

20. Bates SM, Greer IA, Hirsh J, Ginsberg JS. Use of antithrombotic agents during pregnancy. Chest. 2004; 126: 627S-644S.

21. Ramin SM, Ramin KD, Gilstrap LC. Anticoagulants and thrombolytics during pregnancy. Semin Perinatol. 1997; 21: 149-53.

22. Toglia MR, Weg JG. Venous thromboembolism during pregnancy. N Engl J Med. 1996; 335: 108-14.

23. Chunilal SD, Young E, Johnston M. Comparison of the nonespecific binding of a low molecular weight heparin (dalteparin) with unfractionated heparin in pregnant and non-pregnant plasma (abstract). Thromb Haemost. 1999; (Suppl.): 532

24. Wutschert R, Piletta $\mathrm{P}$, Bounameaux H. Adverse skin reactions to low molecular weigh heparins: frequency, management and prevention. Drug Safety. 1999; 20: 515-25.

25. McKenna R, Cole ER, Vasan U. Is warfarin sodium contraindicated in the lactatin mothers? J Pediatr. 1983; 103: 325-7.

26. Department of Health. Report on confidential inquiries into maternal deaths in the United Kingdom 1994-1996. London: HMSO; 1998.

27. Ahearn CS, Hadjiliadis D, Govert JA, Tapson VF. Massive pulmonary embolism during pregnancy succesfully treated with recombinant tissue plasminogen activator. Arch Intern Med. 2002; 162: 1221-7.

28. Ramin SM, Ramin KD, Gilstrap LC. Anticoagulants and thrombolytics during pregnancy. Semin Perinatol. 1997; 21: 149-53.

29. Fitch JCK, Hines RL. Perioperative use of anticoagulants and thrombolytics. Anesthesiol Clin North Am. 1999; 17: 787-97.

30. Narayan H, Cullimore J, Krarup K, Thurston H, MacVicar J, Bolia A. Experience with the cardial inferior vena cava filter as prophylaxis against pulmonary embolism in pregnant women with extensive deep venous thrombosis. $\mathrm{Br}$ J Obstet Gynaecol. 1992; 99: 637-40.

31. Hux CH, Wapner RJ, Chayen B, Rattan P, Jarrell B, Greenfield L. Use of the Greenfield filter for thromboembolic disease in pregnancy. Am J Obstet Gynecol. 1986; 155: 734-7.

32. Richards SR, Barrow J, O'Shaughnessy R. Intrapartum pulmonary embolus. J Reprod Med. 1985; 30: 64-6.

33. ESC Guidelines. Guidelines on the diagnosis and management of acute pulmonary embolism. Eur Heart J. 2008; 29: 2276-315.

Os critérios diagnósticos são: pressão sistólica da artéria pulmonar (PSAP) $\geq$ a $30 \mathrm{mmHg}$ e a diastólica (PDAP) $\geq$ a $15 \mathrm{mmHg}$, pressão média na artéria pulmonar (PMAP) $\geq 25$ $\mathrm{mmHg}$ em repouso, ou $\geq 30 \mathrm{mmHg}$ durante o exercício ${ }^{1,2}$.

\section{2 - Classificação}

A HAP é classificada em três subgrupos:

a) Hipertensão arterial pulmonar idiopática (HAPI).

b) Hipertensão arterial pulmonar familiar (HAPF).

c) Hipertensão arterial pulmonar relacionada a fatores de 